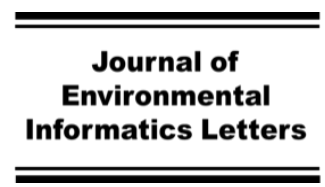

www.iseis.org/jeil

\title{
Computational Analytics for Supporting Environmental Decision-Making and Analysis: An Introduction
}

\author{
J. S. Yeomans ${ }^{1 *}$, Guest Editor \\ ${ }^{I}$ Schulich School of Business, York University, Toronto, Ontario M3J 1P3, Canada
}

Received 19 November 2020; revised 26 November 2020; accepted 13 December 2020; published online 31 December 2020

In practice, environmental informatics involves a combination of computers, computation, mathematical modelling, and system science to address real-world environmental problems. This special issue includes a number of applied computational analytics papers that either create new methods or provide innovative applications of existing methods for assisting with environmental decision-making applications using informatics. In line with the aims and scope of the special issue, the diversity of applications in the papers highlights a wide spectrum of both practical relevance and methodological contributions to research in environmental decision-making and analysis. The contributions contained in this issue all demonstrate novel approaches of computational analytics as applied to environmental decision-making - be this on the side of modelling, computational solution procedures, visual analytics, and/or technologies.

In the first paper, the design of support-mechanisms for renewable energy policy is considered. In order to select desirable support mechanisms, decision-makers need to possess a sound grasp of the most likely outcomes from different policy designs. In Analyzing Renewable Energy Policies - Using the Pay-Off Method to Study the Finnish Auction-Based Renewable Energy Policy, Hietanen, Kozlova, and Collan introduce a fuzzy pay-off-based real options approach for analyzing renewable energy designs for auction-based support mechanisms. This real options approach enables both proper beforeimplementation policy analysis and an analysis of results that is intuitively understandable for the decision-makers. Using a renewable energy case from Finland, they show that their process is relatively straightforward to implement, while producing intuitively understandable information to support the design selection.

In the second paper, Identifying and Characterizing Cyclicality and Seasonality in Environmental Data with an Adaptive

\footnotetext{
*Corresponding author. Tel.: +1 (416) 7365074.

E-mail address: syeomans@schulich.yorku.ca (J. S. Yeomans).

ISSN: 2663-6859 print/2663-6867 online

(C) 2020 ISEIS All rights reserved. doi:10.3808/jeil.202000040.
}

Filter Implementation of a Phase Locked Loop, Linton and Daw examine the use of Phase Locked Loops as an alternative approach for identifying the presence of cycles in environmental data. As the challenge of data overload becomes increasingly prevalent, techniques requiring minimal computing resources that can evaluate relationships gain ever-increasing importance. The speed of Phase Locked Loops makes them ideal for evaluating Big Data and/or data flows that are so large that they must be assessed on a real time basis. In this respect, the Phase Locked Loop is superior to other techniques used to evaluate cyclical behavior in environmental decision-making, engineering, business, and other related fields. The paper applies the Phase Locked Loop approach to weather data.

In Optimizing a Two-Stage Modular Wastewater Plant Expansions Using Numerical Methods and Simulation in a Real Options Context, Lawryshyn, Zhang, and Davison use real options to simultaneously develop (i) a numerical model and (ii) a model based on Monte Carlo simulation, to provide a decision mechanism for optimizing wastewater plant expansion strategies. The optimization of a modular expansion strategy, while extremely relevant in industrial settings, requires sophisticated numerical modeling for the valuation of even the simplest scenarios. The Monte Carlo approach is more intuitive to practitioners and more versatile in that it can model nonstandard processes. Since it is shown that both the numerical and simulation models match consistently, the results support the Monte Carlo approach for practical decision-making purposes.

In System Modeling and Sensitivity Analysis of the Iowa Food-Water-Energy Nexus, Raul, Leifsson, and Kaleita examine the interdependencies of the Iowa food-energy-water (IFEW) system. The state of Iowa is a significant contributor of nitrogen loads throughout the Mississippi river basin. With excessive water flowing through the water system of Iowa, surplus soil nitrogen is transported via the Mississippi river basin into the Gulf of Mexico, resulting in a hypoxic zone causing major detrimental environmental impacts. These nitrogen loads arise from both high yield agriculture and animal agriculture. The IFEW computational model is employed by 
decision-makers to formulate policies that mitigate the adverse impacts of nitrogen exported into the environment and the economy. The analysis reveals that the commercial nitrogen application rates for corn and corn yield are the most critical parameters contributing to the nitrogen surplus.

In River \& Estuary Observation Network: Refinement of Stage Height Sensor Subsystem for Low Cost and High Reliability, Kirkey et al. examine the software and on-site measurements for accurately obtaining water stage data from submersible pressure sensors installed at autonomous stream gauging stations. The system incorporates pressure sensor offset errors, water density, and local gravitational acceleration to produce a stage height reading. A custom-designed, low-cost, submersible pressure sensor is introduced that exhibits minimal errors without the need for sensor calibration. These advances, when integrated into previously developed inexpensive base stations, permit the accurate monitoring of stage height at remote locations with low installation and operating costs.

In the paper Implications of Pulp and Paper Mill Primary Sludge Incineration Versus Use in Composites Production: Decision-Making Based on Life Cycle Assessment, Mustonen et al. use a lifecycle assessment approach to assess the environ-mental decision-making consequences of diverting pulp and paper mill sludge from conventional end-of-life incineration into the production of composites, instead. While primary sludge incineration generates only a small climate change impact, utilization of primary sludge in the production of composites turns out to be a more environmentally friendly option. A sensitivity analysis shows that composite production that replaces plastic is only better when virgin plastic is substituted beyond a particular minimum ratio. Low substitution rates are possible if the properties of the composites are worse than those of virgin plastic, in which case incineration proves the better alternative.

In Visual Analytics in Environmental Decision-Making: A Comparison of Overlay Charts Versus Simulation Decomposition, Kozlova and Yeomans contrast the performance and benefits of two visual analytics tools - overlay charts and simula- tion decomposition - for environmental decision-making. Monte Carlo simulation approaches have frequently been used to incorporate a wide spectrum of uncertainties into environmenttal decision-making. Analytical visualization of the disparate uncertainties is a crucial component for effective decision support in Monte Carlo analysis. Overlay charts enable the display of multiple sources of uncertainty stacked on top of each other in a single graphical representation and come as a standard feature in numerous commercial Monte Carlo software packages. Conversely, simulation decomposition is a new analytical technique that combines user-defined sub-distributions of the simulation uncertainties and collectively displays them in a combined graphical output figure. This paper contrasts the efficacy of overlay charts versus simulation decomposition for the visual analysis of uncertainty in the environmental decisionmaking process.

In the final paper, Integrating Societal and Scientific Elements into Sustainable and Effective Water Resource Policy Development, Gutenson et al. provide their Water Resources Integrated Science and Policy Research (WRISPR) to successfully integrate stakeholders and policy makers into the scientific process of water policy formation using a framework built upon both cyberinfrastructure and modeling tools. The various scientific areas that encompass the "water disciplines" are as diverse as the numerous stakeholder standpoints. Thus, it is essential for scientists, engineers, and policy makers to assimilate all aspects of water resources management into the decision-making process. It is demonstrated that WRISPR can be scaled to reinforce science-based water policy and decisionmaking over multiple spatial and temporal ranges.

As guest editor, I am extremely grateful to all of the authors and reviewers for their valuable contributions and forthright cooperation in completing this special issue. I greatly appreciate the opportunity to have participated in all aspects of the project and would like to thank all editorial members affiliated with the Journal of Environmental Informatics Letters and ISEIS for their ongoing support and encouragement. 